\title{
Comparing the Interpretation of Traumatic Chest X-Ray by Emergency Medicine Specialists and Radiologists
}

\author{
Saeed Safari ${ }^{1}$; Alireza Baratloo ${ }^{1,{ }^{*}}$; Ahmed Said Negida ${ }^{2}$; Morteza Sanei Taheri ${ }^{3}$; Behrooz \\ Hashemi ${ }^{1}$; Samaneh Hosseini Selkisari ${ }^{4}$ \\ ${ }^{1}$ Department of Emergency Medicine, Shohadaye Tajrish Hospital, Shahid Beheshti University of Medical Sciences, Tehran, IR Iran \\ ${ }_{3}^{2}$ Faculty of Medicine, Zagazig University of Medical Sciences, Zagazig, Egypt \\ ${ }^{3}$ Department of Radiology, Shohadaye Tajrish Hospital, Shahid Beheshti University of Medical Sciences, Tehran, IR Iran \\ ${ }^{4}$ Department of Medicine, Shahid Beheshti University of Medical Sciences, Tehran, IR Iran \\ *Corresponding author: Alireza Baratloo, Department of Emergency Medicine, Shohadaye Tajrish Hospital, Shahid Beheshti University of Medical Sciences, Tehran, IR Iran. Tel: +98- \\ 9122884364, Fax: +98-2122721155, E-mail: alirezabaratloo@yahoo.com
}

Received: July 20, 2014; Revised: August 8, 2014; Accepted: September 22, 2014

Background: Discrepancy between X-ray readings of emergency physicians (EPs) versus radiologists was reported between 0.95\% and $16.8 \%$ in different studies. The discordance was even higher when specific studies such as chest X-rays (CXR) were probed.

Objectives: This prospective study was conducted to assess the discrepancies between emergency and radiology departments with respect to interpretation of the traumatic chest X-rays.

Patients and Methods: This prospective study was conducted in Shohadaye Tajrish Hospital, Tehran, Iran, from March to April 2014. Based on Advanced Trauma Life Support (ATLS) guidelines, plain chest radiography (CXR) was ordered for all patients in two standard views of posterior-anterior and lateral. All CXRs were interpreted by a corresponding emergency medicine specialist and a radiologist blind to the clinical findings of the patients. Finally, the results of two interpretations were compared. Accuracy, sensitivity, specificity, and predictive values of traumatic CXR interpretation were calculated by EPs with $95 \%$ of confidence interval (CI).

Results: The evaluation of EPs was identical to that of the radiologists in $89.5 \%$ of the cases. Ninety-eight percent (98\%) indicated total agreement and 1.5 percent total disagreement.

Conclusions: There is a high agreement between EPs and radiologists in CXR interpretations in Shohadaye Tajrish Hospital. Thus, EPs can substitute radiologists in the emergency department. More improvements are recommended to achieve the standard level of agreement.

Keywords: Emergency Medicine; Advanced Trauma Life Support Care; Radiography, Thoracic; Radiographic Image Interpretation

\section{Background}

Emergency department (ED) is the critical ward in hospitals. Overcrowding and urgent nature of the patients makes EDs more crucial and turns them to a particularly challenging area. Therefore, the importance of accurate and fast diagnosis needed for a proper management is obvious $(1,2)$.

Traumatic patients make up the major bulk of emergency clients and almost always CXR is the most common investigation being used in their evaluation process. CXR usually requires to be interpreted by an emergency physician (EP) and management plans are initiated before the formal radiologist's interpretation (3). CXR is the most common type of radiograph that is misinterpreted by observers, especially in the ED, nevertheless not so much data are available regarding traumatic patients. Also, the considerable number of ED cases, including traumatic ones present in late evening, night, or weekend hours that access to the radiologist is nearly impossible. Thus, EPs may remain ignorant about the final read- ing and finding their mistakes, which can have impact on patient's care $(4,5)$. The discrepancy rate between X-ray readings of EPs versus radiologists was reported between $0.95 \%$ and $16.8 \%$ in different studies. This discordance was even higher when some specific studies like CXR were probed $(5,6)$, but it did not assess well in traumatic patients. In addition, false radiologic interpretations were described differently in various studies. For instance, while some studies only take false negatives into account, in others both false negative and false positive cases were considered as misinterpretation. Thus, the reports of discrepancy vary between $14 \%$ and $33 \%$ in different studies (2, $6,7)$.

Although CXR is a useful clinical imaging technique in $E D$, especially for the primary evaluation of traumatic patients, the diagnostic accuracy of CXRs still depends on the clinician's experience $(8,9)$. Advancement in techniques and equipment of diagnostic imaging (like digitalization) was made to improve the quality of CXR, but

Copyright (C) 2014, Kashan University of Medical Sciences. This is an open-access article distributed under the terms of the Creative Commons Attribution-NonCommercial 4.0 International License (http://creativecommons.org/licenses/by-nc/4.0/) which permits copy and redistribute the material just in noncommercial usages, provided the original work is properly cited. 
it is too expensive to use in all health-care units. Since interpretation accuracy of traumatic CXR by EPs is vital, improvements in the diagnostic accuracy of CXR by developing the interpretation's technique could be more beneficial than technical improvements in the quality of CXRs $(8,10)$.

\section{Objectives}

We conducted a prospective study to assess the discrepancies between emergency and radiology departments in the interpretation of CXRs.

\section{Patients and Methods}

This prospective study was conducted in the ED of Shohadaye Tajrish Hospital, Tehran, Iran, from March to April 2014. The study population comprised of patients referred to the trauma unit following multiple traumas. The study protocol was approved by Ethics Committee of Shahid Beheshti University of Medical Sciences and the informed consent form was fulfilled by the participants. Based on the advanced trauma life support (ATLS) guidelines, CXR was ordered for all patients in two standard views of posterior-anterior and lateral. Based on this method, we wanted to compare the mentioned skill by interpretation of posterior-anterior (PA) and lateral views, however, it is impossible or useless to obtain these views in patients who are hemodynamically unstable, have depressed level of consciousness, or flail chest. In these patients, an anterior-posterior (AP) portable chest was the alternative choice and sometimes chest CT may substitute PA and lateral views during their management process. Consequently, patients with hemodynamic instability, depressed level of consciousness, and flail chest were excluded.

All CXRs were interpreted by a corresponding emergency medicine specialist and the results were submitted in a specific data gathering form. Positive CXRs were considered as ones with any of the following findings: pneumothorax; hemothorax; dislocation rib; sternum, scapular, or clavicle fracture; wide mediastinum lung contusion; and subcutaneous emphysema. All CXRs were interpreted again by a radiologist blind to the clinical findings of patients. Finally, the two interpretations were compared. Accuracy, sensitivity, specificity, and predictive values of traumatic CXR interpretation were calculated by an EP with 95\% confidence interval (CI).

\subsection{Statistical Method}

The data were entered into the Microsoft Excel sheet. Statistical analysis was performed using MedCalc software. Descriptive statistics was generated using chi-square test to assay the difference in evaluation proportions. The proportion of agreement (po) in each diagnostic item was calculated as the percentage between the sum of correct evaluations and the total evaluations. Bland-Altman plot was used to indicate the upper and lower limits of agreement. P value $\leq 0.05$ was considered statistically significant.

\section{Results}

Of 105 patients, 82 were male and 23 female. Their ages ranged from 1 to 75 years $(33.1 \pm 14.1)$. The most common physical examination findings were as follows: destructive injury (92.4\%), abnormal chest exam (21\%), local chest pain (17.1\%), and shoulder tenderness (8.6\%).

The EP evaluation was identical to that of the radiologist in $89.5 \%$ of the cases. The total proportion of agreement was $98.5 \%$ and disagreement was $1.5 \%$. The proportion of agreement in each item is shown in Table 1 and Figure 1.

The upper marker indicates the least agreement, 92.4\%, achieved in normal chest and the lower marker shows the highest agreement, $100 \%$, in cases with cardiomegaly, wide mediastinum, sternal fracture, and dislocation. Sensitivity, specificity, positive predictive value (PPV), and negative predictive value (NPV) of EP in traumatic CXR interpretation were 100\% (95\% CI: 62.91-100.00), 100 (95\% CI: 96.23-100), 100\% (95\% CI: 62.91-100), and $100 \%$ (95\% CI: 96.23-100), respectively.

\begin{tabular}{lcccc}
\hline Table 1. Details of Agreement Proportion & \multicolumn{3}{l}{} \\
\hline Cases/With & $\mathbf{X}^{\mathbf{2}}$ & P value & Agreement, \% & Disagreement, \% \\
\hline Normal cases & 91.273 & $\mathrm{P}<0.0001$ & 92.4 & 7.6 \\
\hline Hemothorax & 25.748 & $\mathrm{P}<0.0001$ & 99.0 & 1.0 \\
\hline Pneumothorax & 58.300 & $\mathrm{P}<0.0001$ & 98.1 & 1.9 \\
\hline Lung contusion & 58.300 & $\mathrm{P}<0.0001$ & 98.1 & 1.9 \\
\hline Scapular fracture & 25.748 & $\mathrm{P}<0.0001$ & 99.0 & 1.0 \\
\hline Clavicle fracture & 58.300 & $\mathrm{P}<0.0001$ & 98.1 & 1.9 \\
\hline One-rib fracture & 25.748 & $\mathrm{P}<0.0001$ & 99.0 & 1.0 \\
\hline Two-rib fracture & 72.061 & $\mathrm{P}<0.0001$ & 97.1 & 2.9 \\
\hline Sternal fracture & identical & - & 100 & 0 \\
Wide mediastinum & identical & - & 100 & 0
\end{tabular}


Safari S et al.

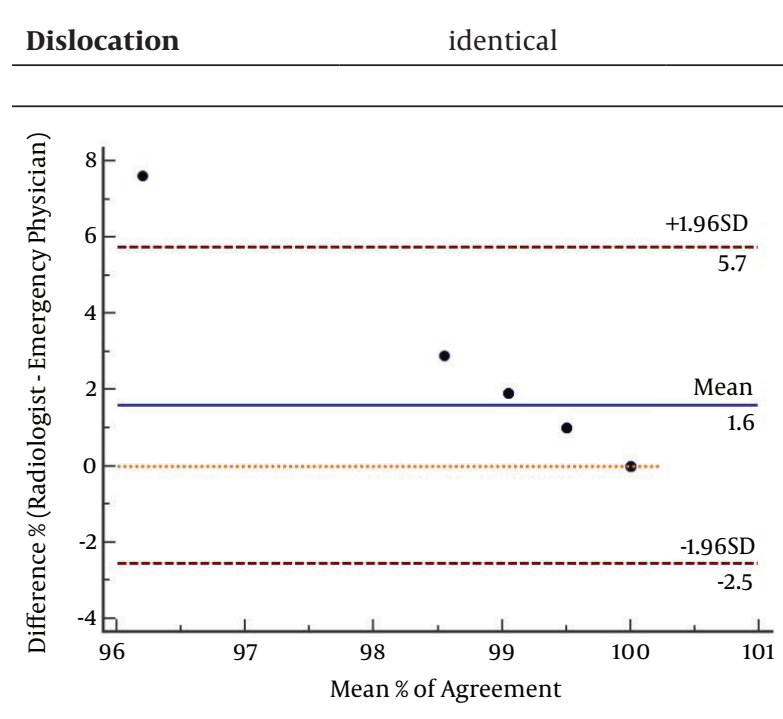

Figure 1. Differences in Interpretations Between the Two Raters is Plotted Against the Mean \% of Agreement in Bland-Altman Plot

\section{Discussion}

The results of the current study showed that CXR interpretation of EPs for traumatic patients had acceptable sensitivity and specificity in comparison to the radiologists' interpretation.

Our study was targeting a real challenge in most of emergency units of health institutes. Based on the increase in the interest of raising the quality of healthcare and providing the best care for the patients as well as guidelines of Joint Commission on Accreditation of Health Care Organization (JCAHO), CXR should be read by a radiologist to achieve the most accurate interpretation. EPs' skills have been examined in different radiology studies $(1,3)$. EPs frequently miss specific radiographic abnormalities, consequently there is a considerable discrepancy between their interpretations and those of the trained radiologists (5). Interestingly, reports of discrepancy of as high as $13.2 \%$ can be found in the literature about the two radiologists reading the same radiology study (1). False negatives were the potential harm, led to miss the patient with life-threatening condition. On the other hand, our rationale behind taking the false positives was the fact that these diagnoses would warrant further investigations and longer hospitalization; also it could subsequently inflict unnecessary financial burden on the patient and health system, not to mention the overcrowding of ED by delaying the diagnosis and discharge process (1). Studies revealed that the interpretation of patients' CXR by a radiologist improves the quality of diagnosis, especially regarding complicated images. Because radiologist is not available in the ED at overnight during the week, EPs should interpret the findings, especially for urgent cases without waiting for the attendance of a radiologist. Numerous studies have examined the interobserver reli- ability of radiographic interpretations in the ED. Since each study was designed in a different way, the standardization would be difficult $(1,7,11-13)$.

Studies showed that misdiagnosis is common among EPs' interpretations. Furthermore, not reporting the findings may lead to serious complications and adverse effects (1), which contradicts the healthcare quality.

As a part of healthcare system, we believe that it is important to compare the CXR interpretations of both emergency and radiology physicians to find out how much discrepancy is present. This will lead to the next step of decision making, and finding a solution for this problem.

The results of the present study revealed that there is an overall discordance of $1.5 \%$ between EPs' and radiologists' CXR interpretations. According to Gatt et al. (5) the discordance between radiologist and EP interpretations should be $0.3 \%$ or less to be considered as nonsignificant. As a result, the discordance measured in our study is clinically significant, which means that more improvements are needed to raise this level of agreement. The most relevant misinterpreted items were normal chest, two-rib fracture, clavicle fracture, contusion, and pneumothorax. Misinterpretation of normal CXR by EPs (false positive) can be accompanied by an unnecessary and a high cost process like CT scan. Another possible reason of such misinterpretation may be due to the presence of distractive findings that can lead to miss all injuries. Presence of such discrepancies indicates further training of EPs in this valuable skill.

In comparison with similar studies in other healthcare institutions, this level of agreement is satisfactory and high enough to allow EPs to interpret CXR alone; although the discrepancy was clearly low, it was still significant.

To discuss these findings, the factors affected the accuracy of such interpretative comparisons should be listed as follow:

1) Quality of the emergency medicine education in the institution,

2) The difficulty level of CXR findings e.g. presence of small size pneumothorax,

3) Degree and experience of the interpreting physicians, 4) The psychological factor of preparedness causes that EPs give more concern and attention to detect all findings in the radiograph.

This high degree of agreement can be initially attributed to the high quality of emergency medicine education in Shohadaye Tajrish Hospital, Tehran, Iran.

Other possible reasons for this degree of agreement are as follows: 1) The radiographs were easy to interpret and not complicated as cases in other studies. A proof supporting for this reason was the baseline characteristics of the included cases that were not so complex. 2) The psycholog- 
ical factor of preparedness in EPs interpreting the radiographs that could be eliminated if the study was designed to compare EPs vs. radiologists. A proof supporting of this factor is that the degree of agreement was least in the normal CXRs (92.4\%). EPs tend to give attention to any possible findings, which make false positive interpretations.

\subsection{The Possible Solutions}

Lucas et al. (14) suggested enhancing the part of radiology in the residency curriculum of emergency medicine, which would enable radiologists to interpret radiographic images perfectly.

Maryland University had a leading experience (15) in developing a network system that allows radiologists to interpret the radiographic findings without a physical presence in the ED. It is also a rapid system that allows radiologists to overlook the preliminary interpretations and comments of Eps, which will supply the missing parts regarding the historical information of the patient and the impression of EPs towards the patient.

Continuous training and weekly courses for EPs are recommended solutions that will enhance their CXR interpretative skills and minimize the possible errors till a solo interpretation, independent from radiologists, can be achieved with less than $0.3 \%$ discordance.

In case of urgent cases, when EPs' interpretation is needed, it is recommended to interpret those CXR images by a senior EP, not a house officer, resident, or nurse. According to Guly, the educational degree was associated with the percentage of misdiagnosis errors (16).

Ghane et al. (8) described a new interpretation method for CXR to detect small size pneumothorax; this method increased the accuracy of EPs interpretations. Indeed, such low cost methods are recommended.

\subsection{Limitations of the Study}

The study did not reveal the effect of the experience or academic degree on the accuracy of CXR interpretations.

There is a high agreement between EPs and radiologists in CXR interpretations in Shohadaye Tajrish Hospital, Tehran, Iran. EPs can substitute radiologists in the ED. Of course, more improvements are needed to achieve the standard level of agreement.

\section{Acknowledgements}

We would like to express our special thanks to the Emergency Department staff of Shohadaye Tajrish Hospital, Tehran, Iran.

\section{Funding/Support}

All authors declared that this study was accomplished without any funding or support and authors were responsible for all expenses. This study was a part of Dr. Samane Hosseini Selkisari's thesis as general physician course at Shahid Beheshti University of Medical Sciences, Tehran, Iran.

\section{References}

1. Arhami Dolatabadi A, Baratloo A, Rouhipour A, Abdalvand A, Hatamabadi H, Forouzanfar M, et al. Interpretation of Computed Tomography of the Head: Emergency Physicians versus Radiologists. Trauma Mon. 2013;18(2):86-9.

2. Eng J, Mysko WK, Weller GE, Renard R, Gitlin JN, Bluemke DA, et al. Interpretation of Emergency Department radiographs: a comparison of emergency medicine physicians with radiologists, residents with faculty, and film with digital display. AJR Am J Roentgenol. 2000;175(5):1233-8.

3. Brealey S, Scally A, Hahn S, Thomas N, Godfrey C, Coomarasamy A. Accuracy of radiographer plain radiograph reporting in clinical practice: a meta-analysis. Clin Radiol. 2005;60(2):232-41.

4. Petinaux B, Bhat R, Boniface K, Aristizabal J. Accuracy of radiographic readings in the emergency department. Am JEmerg Med. 2011;29(1):18-25.

5. Gatt ME, Spectre G, Paltiel O, Hiller N, Stalnikowicz R. Chest radiographs in the emergency department: is the radiologist really necessary? Postgrad Med J. 2003;79(930):214-7.

6. Gratton MC, Salomone JA, 3rd., Watson WA. Clinically significant radiograph misinterpretations at an emergency medicine residency program. Ann Emerg Med.1990;19(5):497-502.

7. Klein EJ, Koenig M, Diekema DS, Winters W. Discordant radiograph interpretation between emergency physicians and radiologists in a pediatric emergency department. Pediatr Emerg Care. 1999;15(4):245-8.

8. Ghane MR, Saburi A, Javadzadeh HR. A recommended method in order to interpret chest x-rays for diagnosing small size pneumothorax. Int J Crit Illn Inj Sci. 2013;3(1):36-9.

9. Kaufman B, Dhar P, O'Neill DK, Leitman B, Fermon CM, Wahlander SB, et al. Chest radiograph interpretation skills of anesthesiologists. J Cardiothorac Vasc Anesth. 2001;15(6):680-3.

10. Salazar AJ, Camacho JC, Aguirre DA. Comparison between different cost devices for digital capture of X-ray films: an image characteristics detection approach. J Digit Imaging. 2012;25(1):91-100.

11. Mayhue FE, Rust DD, Aldag JC, Jenkins AM, Ruthman JC. Accuracy of interpretations of emergency department radiographs: effect of confidence levels. Ann Emerg Med. 1989;18(8):826-30.

12. Brunswick JE, Ilkhanipour K, Seaberg DC, McGill L. Radiographic interpretation in the emergency department. Am J Emerg Med. 1996;14(4):346-8.

13. Al aseri Z. Accuracy of chest radiograph interpretation by emergency physicians. Emerg Radiol. 2009;16(2):111-4.

14. Lucas R, Choudhri T, Roche C, Ranniger C, Greenberg L. Developing a curriculum for emergency medicine residency orientation programs. J Emerg Med. 2014;46(5):701-5.

15. Siegel E, Groleau G, Reiner B, Stair T. Computerized follow-up of discrepancies in image interpretation between emergency and radiology departments. J Digit Imaging. 1998;11(3 Suppl 1):18-20.

16. Wakai A. Diagnostic errors in an accident and emergency department. Emerg Med J. 2002;19(4):374. 